\title{
Case Study of Healing by the Tran Message of Body for ADHD
}

\author{
Mee-sook Cheong1), Yeon-jung Tae2)
}

\begin{abstract}
This is a narrative case study on the treatment of Attention Deficit Hyperactivity Disorder(ADHD), which occurs when the body is out of balance, through Tran Message of body, which is healing through the balance of the body. The necessity of research is that the disease comes when the balance of the body is broken, so the study of healing through the balance of the body is urgently needed. In order to solve the problem, it is necessary to study self-healing in order to recognize physical, psychological, and mental and environmental problems, and to educate and restore the inherent potential. The purpose of this study is to help the pain and healing of children with ADHD disorder. Through The Tran Message of body healing, it was confirmed that attention deficit hyperactivity disorder of 5 and 6 year old children came from anxiety. We may have to think that it was difficult to breathe because of anxiety and that we had been overactive by taking a rough breath all over our body. It was also found that empathy in humans has the power to tear down even the thickest and hardest parts. The two children struggled, but no one knew the pain of the child, but only scolded and condemned. Empathy was a healing energy for two children. In conclusion, through The Tran Message of body healing, it can be seen that although the diseases are the same, the causes are different and the healing principles are also different. The Tran Message of body healing can be said to be a guide of life that guides how to do for the restoration of humanity and life, which is the task of mankind through obstacles. Since there are only 2 participant, I think more research is needed.
\end{abstract}

Keywords: Tran Massage of Body, Healing, ADHD, Trauma, Stored Information

\section{Introduction}

The human consists of three bodies[1], the visible physical body, the bodies of the invisible mind, and the underlying spirit. Human diseases are manifested in mental, psychological and spiritual diseases as well as in visible physical diseases. As such, the three body's broken, unbalanced disorder symptoms are seen as a disease. It is said tha thealing of disease also has to be accompanied by healing of the visible body, mind and spirit so that the three bodies are balanced and can recover[1].

Received(October 31, 2019), Review Result(1st: November 27, 2019, 2nd: January 04, 2020), Accepted(March 25, 2020)

1) (Professor, Corresponding Author) 02838 Dept. Naturopathy, Dongbang Culture University, Seongbuk-dong, Seongbuk-gu, Seoul, Korea email: ayurveda16@naver.com

2) (Ph. D. Course) 02838 Dept. Naturopathy, Dongbang Culture Graduate University, Seongbuk-dong, Seongbuk-gu, Seoul, Republic of Korea email: tyoj5358@naver.com 
Recently, there are emerging natural healing therapies for diseases that are difficult to cure by modern medicine. One of these methods is the Tran Message of body[2], which is a unique energy of a person to automatically identify a disease upon meeting the person. The healer will move his or her hand moves on the affected area, diagnose the disease, and empathize with it. The symptom revealed by the disease is the ability to search for any energy disorder. It can also recognize information of what pain it is, what causes it, and through what method it is healed. Therefore, we researchers make our clients recognize the physical, psychological, mental and environmental problems, educate them and help them self-heal by recovering their inherent potential.

This is a case study of children with ADHD. It is also a narrative study that expresses each subject well and describes them vividly. According to DSM(Diagnostic and Statistical Manual of Mental Disorders)-5, as a part of neuro developmental disorders, ADHD is hyperactivity disord er caused by attention deficit[3][4]. The essential symptoms of ADHD are inattention, hyperactiv ity and impulsiveness, which are revealed by wandering without completing the task, lack of $\mathrm{p}$ atience, poor concentration and disordered behavior[5]. ADHD is revealed not only with very $\mathrm{d}$ istracted and inattentive behavior, but also with impulsive hyperactivity without proper control of one's behavior[6]. ADHD is impulsive and distracted when acting, making it difficult to focu s. Children with this disorder have considerable difficulties in their home and school life becau se they exhibit significantly more restless, fidgety and impulsive behaviors than the children of the same age.

Children with ADHD are characterized not only by low endurance to frustration, but also by irritability and unstable mood[7]. Even though they are not diagnosed with a specific learning disability, their learning and professional performance are impaired. The causes of ADHD are seemed to be caused by a combination of biological and psychosocial factors as neurological disorders occurring in early childhood. ADHD occurs before 12 years of age, and the manifestations of the disorder appear at home, school and at work. It is often accompanied by delays in language, movement, and social development.

The source of attention control problems, a key component of ADHD, is not yet clear. Some studies suggest that $\mathrm{ADHD}$ is a problem of executive control[5], whilesomestudies have suggested that awakening and orienting function are also problematic[8]. On the other hand, it also weighs the control damage of treatment process, another major aspect of executive function[7]. This can be explained by a variety of areas covering aspects of attention concentration. Therefore, neurobiological research activities on ADHD disorders have been focused on metabolic degradation of the frontal lobe region, which is associated with lack of 
executive control, and dysfunction of the parietal lobe region, which is associated with clove disorders[9]. On the other hand, in relation to motor suppression, dysfunction of the interior prefrontal region and striatum is involved. Neuropsychological studies suggest that failure to ignore prominent information is due to dysfunctional frontal meetings[10][11]. These evidences also suggest that ADHD is associated with the dopamine system[12]. Symptoms of ADHD are similar to those of frontal lobe damage, and the frontal lobe is also a region of dopaminergic projection.

Narrative research is a qualitative research method that is conducted in both descriptive and explanatory narrative. Descriptive narrative is to meaningfully and correctly describe the occurrence and result of an event in life or organization. Through this narrative this study intends to provide self-identity from the individual story used by the story-based speaker's behavioral information to the individual's whole being, and to reconstruct unity[8] with a life narrative. An explanatory narrative clarifies why an event or situation experienced by human action happened. The results and consequences that happen in people's lives have a cause. It also provides an explanation for the cause and effect of the various events that result. This shows the meaning of the event in the whole story and to understand its cause and context.

In the following two views of narrative research, according to Clandinin[13], within this framework of experience, the focus of narrative inquiry is not only on the individual's experience because the individual's experience is composed, formed, expressed, and executedgiven social, cultural and institutional narratives. The researcher studies the individual's experience in the world, which is a story of living and talking about life, which can be studied by texts that hear, observe, live together, write and interpret. Polkinghorne(1988)[14] understand narrative as a cognitive process that organizes human experience into meaningful episodes based on time and causal relationships. The narrative is defined as a product of the narrative, which is almost the same as the narrative, and the research process is divided into the descriptive narrative and the explanatory narrative as the practice of completely treating or recovering the situation through intervention or treatment[15].

This study followed the concept and application method proposed by Clandinin \& Connelly(2000)[13] which are not only most suitable to understand the individual experience of life but also to refer to the research procedure at the same time. In addition, it used the descriptive narrative and explanatory narrative presented by Polkinghorne(1988)[14] as a way of organizing the results of the study. Disease occurs when the balance of the body is broken. Therefore, it is urgently necessary to study healing through the balance of the body. The purpose of this study is also to help to ease pain of children with ADHD, to heal them. 


\section{Study Method}

\subsection{Study Period}

This study was conducted from September 1, 2018 to November 20, 2018. The counseling period of period of these cases was from November 1, 2018 October 31, 2018, a total of 12 months.

\subsection{Study Participants}

Participants in this study were two males aged five, andsix years old with ADHD. They were continuously treated by modern medicine but shifted to this study's research center because they found the previous ineffective. The 5-year-old child was diagnosed with severe ADHD at S Hospital, and the 6-year-old child was diagnosed with ADHD severe at Hospital D. Since the study participants were all minors, the study was conducted after explaining the contents to the parents and after getting their approval.

\subsection{Data Analysis Method}

This is a narrative research[16] based on subjective interpretive epistemology that studies and interprets the unmanipulated words or behavioral trace of the study participants. As a form of inquiry to understand the human experience, the basic structure of the experience is focused on the understanding of the human and the experience of understanding the human experience prior to understanding the disease that gives meaning to the experience. Narrative research provides a framework for thinking about experience in a three-dimensional space consisted of phenomena, situation, continuity and interaction.

\section{Study Result}

\subsection{Symptoms (Descriptive Narrative)}

The two participants had severe ADHD as indicated by the ir hospital certificate. Unlike ordinary children, they seemed to wander excessively or lack patience or have a short attention or be disordered, because of their inattention, hyperactivity and impulsiveness. The two 
participants did not sit still. Then, while walking around, something broke or spilled.

The five-year old patient, during his first visit in the laboratory, did not sit for a moment and continued to throw anything within reach. At that time, the parent of the child was busy catching him. His parents have been dedicated on his medical needs for many years. However it seems that they have been mentally, physically and economically exhausted because nothing has changed. The parents' frustration reflects on their tiredness. Their child was eating anything he could get on. His staple food was ramen causing the child's obesity. He was unable to hold eye contact with others, and showed the symptom accompanied by an upward rolling of the eyes.

The six year-old child visited the laboratory with his grandmother because his parents were busy. He entered the counselor's office and checked the mood of he healers first, but soon began to frantically run here and there in the lab. Although he had the ability to communicate with his grandmother, his verbal expression level in the language development was much lower than children his age. The child looked obese. When the counselor recommended a snack, he could not settle in one place while eating. Then, he began to eat the other food without focusing on one food. While eating a snack, its crumbs ran out from his mouth, and even while drinking water, the amount of water to run out from mouth was more than to drink.

ADHD is revealed not only with very distracted and inattentive behavior, but also with impulsive hyperactivity without proper control of one's behavior. Because the participants doesn't stay quiet, it was broken or scattered while touching the device around it. The participants showed special cognitive and behavioral problems differed from the behavior of normal children. The descriptive narrative did not draw much of the story because it was a cognitive process that organizes meaningful episodes in the cause-and-effect relationship.

\subsection{Diagnosis (Explanatory Narrative)}

According to the diagnosis of the two participants of this study, they basically have the same problem in the attachment relationship with their parents.

Because the five-year-old was distracted the counselors made him fall into his father's arms. As the child got his breath back and calmed down, they held his hand, let him meditate for a while and then laid him down. As the counselor put her right hand on the child's chest, a warm energy flowed through his chest and he breathed out a few times and then burst into big tears. She allowed him to cry louder and waited until he stopped himself crying. She 
gently pressed his chest with her forefinger again and embraced him with the other hand. To press here is not caused by the counselor's external force, but exerted by the waves of energy which the client's inner side needed. As soon as the child got his breath back, his face began to bloom. The Tran Message of his body showed that the child was shocked from the fetus on and was always in tension. As a result, the Tran Message of his body revealed that because his mother was shocked and surprised in her pregnancy, she did not share her feeling with her son. His mother regarded it as too hard to care for him. Even she always wanted to leave somewhere. Because of anger, the child had something clogged in his chest, which prevented his energy from flowing and have difficulties in breathing. The behavior in which the child tried to live was seen as severe anxiety and hyperactivity. From this case, we could know that the cause of the child's ADHD symptoms was psychological. The child has been unable to discharge waste from the body because the energy in his chest was clogged. Because energy was not circulating through the body adequately enough, normal breathing was not possible. Therefore he tried to maintain breathing through excessive movement. His difficulty in breathing is seen as severe anxiety and hyperactivity. This suggests that the cause of ADHD is psychological.

In the case of the six year-old child, the Tran Message of his body revealed that his anxiety and nervousness was due to his fear that his mother might leave him. According to his grandmother, his parents were separated and his mother had run away from home before separation. On each occasion, she brought him up, so he was emotionally very anxious. As soon as the child fell into his grandmother's arms and the counselors placed their hands on his feet, the child felt easy. This time, the counselor felt that the child had so long put up with crying. She also shed tears of empathy for the child. As the counselor called the child's name and said to him, "You've so long put up with though you've wanted to cry.", he began weeping and crying. As she said to the crying child, "Though you've wanted to cry you could have not cried. You can cry now!", he started crying much louder with overwhelmed emotions. His grandmother cried with him. Then, the crying child soon fell asleep. When he woke up, he drank water, then, took a pee. As the child was more relaxed, the counselor placed him alone in his bed and put her hand on his chest. What the child felt in his whole body was fear and anxiety.

As mentioned above, impulsive behavior was neither intended by the child nor controlled in his head. The child always trembled with fear and behaved harshly to live. In this way the explanatory narrative provides an explanation for the cause and effect of the various events that cause something to happen. 


\subsection{Healing(Curative Narrative)}

The healing was dominated by the recovery of trust with the main caregiver (mother), and this was the way for the child to have faith in the world and to develop his identity.

The five year-old child was so guided with the Family-positioning Program that he could rightly position his parents. When the counselor's hand was led to the child's head, she could feel that his brainwave was not flowing smoothly. The child was unable to smoothly use his energy of the brain in the meantime. However the counselor transmitted him energy with her warm hands so that he could awaken it. Then, as his circulation improved, he was able to breathe normally. Phew!...As the child breathed out, it seemed that the suppressed emotional anxiety disappeared. He soon fell asleep.

\section{"Phew ..." "Phew ..." "The window blow here." "It made my nose open." \\ "Thi ...This(knock chest) is fresh." "Try to touch this!" "Mo ... Mom!"}

Then the counselor put her hand on the child's belly. It seemed that in the meantime, his stomach was filled with gas and was neither digested nor absorbed due to the unbalanced diet. It seemed that his stomach was bulging with gas and was neither digested nor absorbed, which resulted from the unbalanced diet in the meantime. As soon as she touched his stomach with her hands, his stomach began to growl. As his breathing was deeper, he screamed at the top of his lungs. After the five year old had vomited his staple food, ramen, he was able to eat a common meal he never tried before. As he was able to deeply breathe, his distraction and anxiety decreased, too. As the anxiety disorder disappeared, hyperactivity was not only normalized but the eating disorder was also resolved.

$$
\text { "Ah - Ah " "Ah - Ah - !" "I fart." "Wau-dh!" }
$$

The grandmother hugged the six year old who had frantically run here and there with a bite of sweets. The counselor swept and massaged his chest. Then, he soon fell asleep. The child woke up and made a very relaxed face. As the number of the healing by Tran Message of body progressed, he seemed to recognize many concepts (objects, environment, language, food) that he had never had before. Therefore, at first, the counselor led him to behave appropriately according to the development stage of about three years old and praised him for the behaviors. When he wanted to play in the session eight, he started receiving permission 
from the adults around him. In the ninth session, he began to eat foods one by one. In addition, as he could communicate with his mother, he began to speak the language, too.

"I like Mom." "I like Dad." "I love Mom" "Give me a hug!"

“Oh. Good!" "What's this??" "Mom, eat it!" "It's warm"

Through this study, we could know that although the disease was the same, the cause, the symptoms and the healing principle were also different. As well, the trauma of each client could be the appropriate healing principle.

\section{Conclusion and Consideration}

This study is a case of healing participants with ADHD by the Tran Message of body. It is also a narrative research to vividly describe the cause, diagnosis of disorders and healing. Through the Tran Message of body, we confirmed that ADHD of 5 and 6 year old children resulted from anxiety. We have to think about the fact that they had difficulty in breathing because of anxiety, and that they breathed roughly with all their strength just to live, and so acted excessively. In addition, we could also find that empathy gave humans the power to tear down even the thickest and hardest parts. In fact, the two children struggled hard. However no one knew the pain of the children, only scolding and condemning them. Indeed empathy was a healing energy for two children. With doctors, same disease usually diagnosed and prescribed with the same medical needs. In healing by the Tran Message of body, the method is individualized, and the prescription is customized despite same type of disease.

In conclusion, the healing by the Tran Message of body can be said to be a guide of life that shows how we should recover humanity and life from obstacles. One of the limitations of this study is it minimal cases of evidence on the healing process. The Tran Massage of the body finds out what the client cannot say with words, but only with gestures. And they get closer, form attachment, understand, and empathize. The unfortunate thing in this study was that it was difficult for participants to self-express and it was difficult to talk vividly and talk again. And because of the size of the paper, I'm sorry to make an abbreviated announcement the case. And since there are only 2 participant, I think more research is needed. 


\section{References}

[1] M. S. Cheong, Theory and Practice of Ayurveda, Yoerai, (2017), pp.128-129.

[2] Y. J. Tae, 'TRAN THE MESSAGE' heals me, Tarea, (2015), p.23.

[3] U. K. Lee, Abnormal Psychology - DSM-5 update-, hakjisa, (2017), pp.59-66.

[4] S. M. Kwon, Basics of Abnormal Psychology, hakjisa, (2014), p.378.

[5] R. A. Barkley, Attention-Deficit Hyperactivity Disorder, Guilford, (1998)

[6] J. Y. Choi, G. Park, H. H. Seo, Abnormal Psychology, hakjisa, (2016), pp.447-448.

[7] J. T. Nigg, Is ADHD a disinhibitory disorder?, Psychological Bulletin, (2001), Vol.127, No.5, pp.571-598, DOI: $10.1037 / 0033-2909.127 .5 .571$

[8] J. M. Swanson, J. A. Sergeant, E. Taylor, E. J. S. Sonuga-Barke, P. S. Jensen, D. P. Cantwell, Attention-deficit hyperactivity disorder and hyperkinetic disordoer, The Lancet, (1998), Vol.351, No.9100, pp.429-433, DOI: 10.1016/S0140-6736(97)11450-7

[9] K. G. Sieg, G. R. Gaffney, D. F. Preston, J. A. Hellings, SPECT brain imaging abnormalities in attention deficit hyperactivity disorder, Clinical Nuclear Medicine, (1995), Vol.20, No.1, pp.55-60, DOI: 10.1097/00003072-199501000-00014

[10] K. Rubia, S. Overmeyers, E. Taylor, M. Brammer, S. C. R. Williams, A. Simmons, E. T. Bullmore, Hypofrontality if in attention deficit hyperactivity disorder during higher-order motor control: a study with functional MRI, American Journal of Psychiatry, (1999), Vol.156, No.6, pp.891-896, DOI: 10.1176/AJP.156.6.891

[11] G. Bush, J. A. Frazier, S. L. Rauch, L. Seidman, P. J. Whalen, M. A. Jenike, B. Roson, J. Biederman, Anterior cingulate cortex dysfunction in attention- deficit/hyperactivity disorder revealed by FMRI and the Counting Stroop, Biological Psychiatry, (1999), Vol.45, No.12, pp.1542-1552, DOI: 10.1016/s0006-3223 (99)00083-9

[12] J. Y. Choi, G. Park, H. H. Seo, Abnormal Psychology, hakjisa, (2016), pp.447-448.

[13] D, J. Clandinin, Engaging in Narrative Inquiry, Routledge, (2013)

[14] D. E. Polkinghorne, Narrative Knowing and the Human Sciences, State University of New York Press, (1988)

[15] W. H. Chang, Healing Experiences of Middle-Aged Women from Dysfunctional Families: A Meditation Counselling based Narrative Study, Dongbang Culture University, Ph. D. Thesis, (2018)

[16] J. S. Yeom, Narrative Inquiry in Educational Research: The Concept, Procedure, and Dilemmas, Korean Journal of Anthropology of Education, (2003), Vol.6, No.1, pp.119-140. 